\section{MRS Bulletin Volume Organizers guide technical theme topics for 2016}

\author{
www.mrs.org/bulletin
}

$\mathrm{T}_{\mathrm{i}, \mathrm{h}}^{\mathrm{h}}$ he MRS Bulletin 2016 Volume Organizers, who will guide the development of theme topics for the 2016 volume year, are Ilke Arslan (Pacific Northwest National Laboratory, USA), Richard R. Barto Jr. (Lockheed Martin Advanced Technology Laboratories, USA), Boaz Pokroy (Technion-Israel Institute of Technology, Israel), and Zhiwei Shan (Xi'an Jiaotong University, China). Requests for instructions on submitting proposals for MRS Bulletin theme topics can be emailed to bulletin@mrs.org.

Ilke Arslan is a senior scientist at the Pacific Northwest National Laboratory (PNNL) in Washington, USA. She

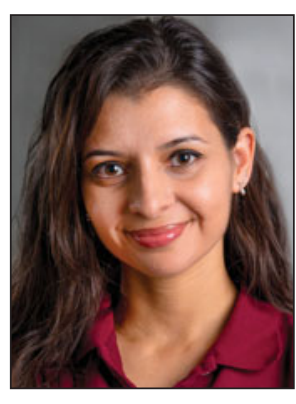
received her $\mathrm{PhD}$ degree in physics from the University of CaliforniaDavis, where she was a member of the faculty of the Chemical Engineering and Materials Science Department before joining PNNL. She has held fellowships from the National Science Foundation and the Royal Society, as well as the Truman Fellowship at Sandia National Laboratories. She has been honored with the Presidential Early Career Award for Scientists and Engineers by President Obama, and was also honored with the Kavli Frontiers of Science Program at the National Academy of Sciences. Arslan's interests include understanding the structure-property relationships of a range of nanomaterials, including catalysts and energy materials, technique development in electron and atom probe tomography, and imaging under liquid and gas environments in the scanning transmission electron microscope.

Richard R. Barto Jr. is senior manager of the Applied Science Laboratory at the Lockheed Martin Advanced Technology
Laboratories in New Jersey, USA, and acting Research Area Manager for Material and Process Technologies. He

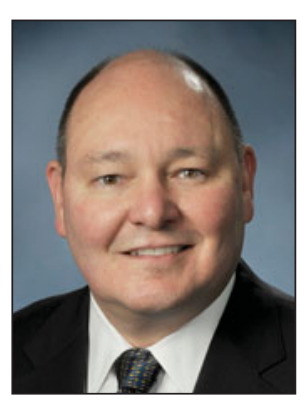

has served as the deputy director for the Lockheed Martin Corporate Advanced Materials and Nanotechnology Initiative, leading the computational modeling and simulation research thrust. He earned a BS degree in chemical engineering from the University of Delaware in 1983, a MS degree in materials science and engineering from Stanford University in 1998 , and a PhD degree in materials science and engineering from Stanford University in 2004. Barto previously worked at the Lockheed Martin Space Systems Advanced Technology Center, where he was a key member of a research team developing wideband nonlinear optical polymer modulators for RF communications, and later as principal investigator for research on tailorable materials for spacecraft thermal and optical control coatings. He joined Lockheed Missiles and Space Company as a composite materials engineer, developing engineering requirements for structural composites for airframes.

Boaz Pokroy is an associate professor in the Department of Materials Science and Engineering at the Technion-Israel Institute of Technology, Israel, where he

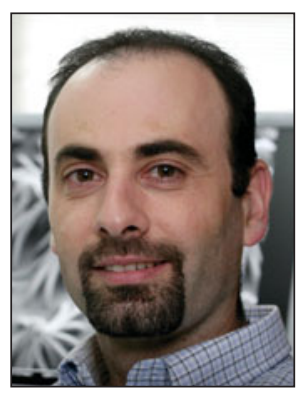
earned all of his degrees. Previously, he was a postdoctoral fellow and F u 1 b r i g h t Scholar in the laboratory of Joanna Aizenberg at the
School of Engineering and Applied Sciences at Harvard University and Bell Labs. Pokroy's research focuses on biomineralization and bioinspired surface engineering. He studies the structure of biominerals on the atomic, nano-, and mesoscales using state-of-the-art highresolution characterization techniques such as high-resolution synchrotron diffraction and aberration-corrected transmission electron microscopy. Based on the strategies that organisms use to produce natural materials, his lab also develops novel bioinspired materials, such as semiconductors whose bandgap can be tuned by the incorporation of intracrystalline biological molecules; controlling the short-range order of nano-amorphous materials; and fabrication of superhydrophobic/superoleophobic surfaces for various applications.

Zhiwei Shan is the Changjiang Professor and Associate Dean of the School of Materials Science and Engi-

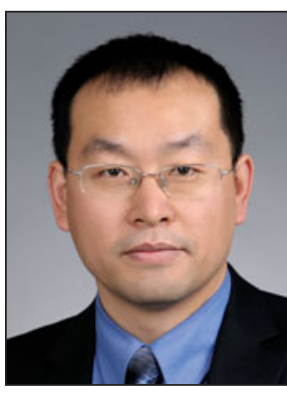
neering at Xi'an Jiaotong University, China. He also holds a joint appointment at the State Key Laboratory for Mechanical Behavior of Materials as the deputy director. He received his bachelor's degree from Jilin University, his master's degree from the Institute of Metal Research, Chinese Academy of Sciences, and his $\mathrm{PhD}$ degree from the University of Pittsburgh. Following his postdoctoral fellowship at the National Center for Electron Microscopy, Lawrence Berkeley National Laboratory, Shan joined Hysitron, Inc. as a product line manager to develop the most advanced in situ TEM and SEM mechanical testing systems. His research interests focus on applying and developing unique quantitative in situ electron microscopy techniques and revealing the novel properties of micro-nanoscaled materials. He has delivered more than 50 invited talks and has authored or coauthored more than 50 technical papers in peer-reviewed journals, including Science, Nature, Nature Materials, and Proceedings of the National Academy of Sciences. 